\title{
Structural Compliance Effects on the Accuracy and Safety of a R-CUBE Haptic Device
}

\author{
G. Carbone ${ }^{1}$, A. Acinapura ${ }^{1}$, D. Mundo ${ }^{1}$, I. Gorgulu ${ }^{2}$, M.I. Can Dede ${ }^{2}$ \\ ${ }^{1} D I M E G$, University of Calabria, Rende, Italy, \\ giuseppe.carbone@unical.it \\ ${ }^{2}$ Izmir Institute of Technology, Izmir, Turkey, \\ candede@iyte.edu.tr
}

\begin{abstract}
This paper addresses the contribution of structural compliance on stiffness and safety of a R-CUBE Haptic Device. Structural compliance is determined in several poses via FEM analysis and addressed by referring to local and global indices of performance. Results are also compared with evidences from experimental tests. Comparison of numerical and experimental data allows to identify and separate the contributions to the overall compliance that are due to the structural stiffness, and other contributions such as joint clearance, pose and loading conditions.
\end{abstract}

Keywords: Parallel Robots, Haptic Devices, Stiffness, Safety

\section{Introduction}

Haptic feedback is mostly combining force or contact feedback to the position information so that users can push, pull, feel, and manipulate objects in virtual environments instead of just seeing a video representation, [1,2]. Over the last decades, a variety of haptic interfaces have been developed ranging from simple single-DOF (Degrees of Freedom) devices to complex, multi-DOF wearable devices often with a trade-off between complexity/workspace and user-friendliness/costs. A haptic device architecture is also affected by the intended application, which can range from augmentation of graphical user interfaces (GUIs) to master interfaces in teleoperation or rehabilitation [1,2]. The PHANToM desktop haptic interface is probably the most commonly used commercial haptic interface, [3].

In a haptic interaction, the position of the end-effector must be measured to generate an accurate haptic stimulus. However, compliant displacements can significantly affect the positioning accuracy, due to the loading condition/poses. Correspondingly, force/torque feedback to the user is generated inaccurately. These effects might become critical also from a safety viewpoint in applications such as the teleoperation of a robot for surgery tasks [2]. However, safety aspects are still needing in-depth investigation as available literature and standards such as [4-6] still do not provide proper 
guidelines for these specific applications. In fact, they mostly aim at eliminating avoidable hazards or using adequate protections to prevent risks, which is often not implementable for haptic devices where a close interaction with human is mandatory. Hence, safety aspects should be here conceived as implementing actions that can reduce and/or mitigate risks, especially from robot mechanical design viewpoint.

In this paper, we limit our attention to the contribution of the structural compliance of a haptic device to both accuracy and safety. Considering rigid joints and no control effects is herewith assumed as the worst-case scenario. We address our attention to the specific case of study of a R-CUBE haptic device prototype. In particular, section 2 describes the R-CUBE architecture; section 3 investigates the structural compliance in several poses via Finite Element Analysis (FEM). Local and global indices of performance are computed and discussed. Results are compared with evidences from experimental tests. The comparison of numerical and experimental results allows identifying and splitting the contributions to the overall compliance that are due to the structural stiffness from the joint clearance or due to the pose and loading conditions.

\section{R-CUBE Architecture}

In the last decades, research on parallel manipulators has been quite rich due to their expected advantages, for example, in terms of stiffness, accuracy, dynamics, as compared with serial manipulators. However, most of these manipulators have coupled motions between the position and orientation of the end-effector as well as they have complex control features also due to singularities. Recent research on parallel manipulators with a reduced number of degrees of freedom (DOFs) has been aiming at possibilities of achieving kinematic architectures with reduction of the number of active DOFs and decoupling of the position and orientation of the end-effector, [7-9].

R-CUBE manipulator is a 3 DOFs parallel manipulator, whose kinematic scheme can be modeled as proposed for example in the scheme of Fig.1.

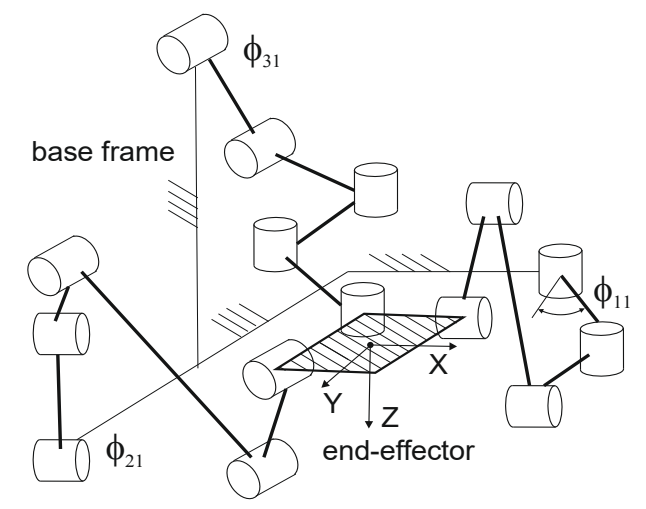

Fig. 1. A kinematic sketch of the R-CUBE manipulator. 
One of its main features is the possibility to achieve decoupled translational motions along $\mathrm{X}-, \mathrm{Y}$ - and $\mathrm{Z}$-axes, by rotating the first joint angle of each chain $\left(\phi_{11}, \phi_{21}\right.$ and $\phi_{31}$ in Fig.1). It is composed of 3 kinematic chains that connect the endeffector to the base frame. Each kinematic chain has 4 links and 5 revolute joints as shown in the scheme of Fig.1. All the actuators can be conveniently located at the base frame. Singularity analysis shows that the singularities can be easily avoided by means of a careful design [9]. The above features make R-CUBE architecture suitable as haptic device. Further details on R-CUBE can be found at [7-10].

\section{$3 \quad$ Structural Performance}

Several attempts have been made to properly model stiffness performance such as reported in $[10,11]$. A stiffness model may be implemented for predicting and compensating compliant displacements on the end-effector for achieving a more accurate and safe haptic control. However, several aspects contribute to the overall stiffness behavior including structural stiffness, joint clearance, joints/motor stiffness, loading conditions and pose of the manipulator.

In this section, we focus on the structural stiffness. The calculation of the structural stiffness is assessed through linear FE-based static simulations within a commercial package. The FE model of the R-CUBE manipulator is created by discretizing the geometry of links and joints as a connected set of four-sided solid elements with four grid points as shown in Fig.2. The aim of the static simulations is to calculate the displacement of the center of gravity of the end-effector (mobile platform). The FE mesh density needs to be properly set up for reducing the overall size of the FE model and, thus, the required computational cost. In particular, the dimension of the discrete elements is here set up to decrease its size going from the base to the end-effector. This choice is made since the displacements are expected to grow accordingly.

Auxiliary rigid body elements are used in order to join the mating links of each chain, obtaining a decoupling of the structural and joints stiffness. The associated material is assumed as a Poly-Lactic Acid (PLA) with specifications from a commonly used material for $3 \mathrm{D}$ printing. The material properties have been obtained experimentally by means of a bending test on a single 3D printed link. Table 1 shows the relationship between the applied bending force $\Delta \mathrm{F}$ and the produced compliant displacement $\Delta \mathrm{x}$ that has been experimentally measured. The stiffness of a link can be estimated as

$$
k=\frac{\Delta \mathrm{F}}{\Delta \mathrm{x}}
$$

On the other hand, the bending stiffness of the link is defined as $[11,12]$

$$
k=3 E I / L^{3}
$$


where $\mathrm{E}$ is the Young Modulus of the material, $\mathrm{L}$ is the length of the tested link and

$$
I=0.15 \frac{\pi D^{4}}{64}
$$

where $\mathrm{D}$ is the diameter of the examined link. The second moment of area $[11,12]$ is considered at $15 \%$ as the used infill value for the 3D printed element with PLA. Hence, knowing that $\mathrm{D}=6 \mathrm{~mm}$ and $\mathrm{L}=129.6 \mathrm{~mm}$ for the tested link and combining equations (1) and (2), it is possible to compute the Young Modulus as E $=2.7 \mathrm{GPa}$.

The base of the R-CUBE manipulator is fixed to the ground while the force is applied at the midpoint of end-effector. The direction of the load is along one of the X-, $\mathrm{Y}$ - and Z-axes according to the considered analysis case. The aim of the simulations is to compute the compliant displacement of the center of the end-effector along the direction of the applied load. The static analysis tests estimate the compliant displacements $(\Delta x, \Delta y, \Delta z)$ of the center of the end-effector. Two reference configurations (Fig. 2) have been chosen to perform the static simulation tests. The simulation results are shown in Table 2 for two different loading conditions. Namely, it has been considered the case with only gravity and the case gravity plus external load of $1.8 \mathrm{~N}$ that is applied along X-, Y- or Z-directions. Results allow to analyze the influence of the own weight of the structure on the overall results. The results show that the effect of the own weight of the structure is not very significant with a maximum contribution of about $18 \%$ along Z-axis. Numerical simulations have been also performed to estimate the main structural eigenvalues that have been chosen as a local stiffness index for the structure, such as proposed in [11].

Table 1. Results of bending tests on a single link with PLA infill equal to $15 \%$.

$$
\begin{array}{|l|llllll}
\mathrm{F}[\mathrm{N}] & 0.34 & 1.32 & 2.30 & 3.29 & 4.27 & 5.25 \\
\mathrm{X}[\mathrm{mm}] & 0.29 & 0.72 & 1.25 & 1.79 & 2.35 & 2.91
\end{array} \mid
$$

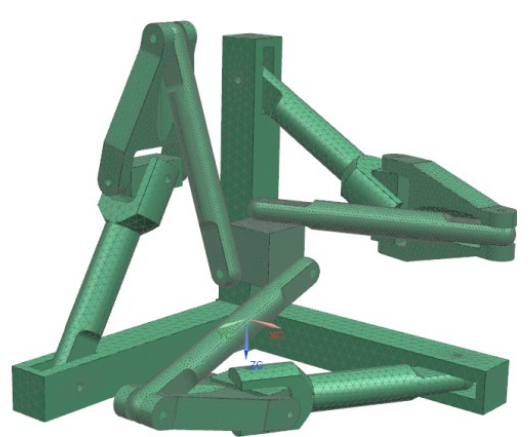

a)

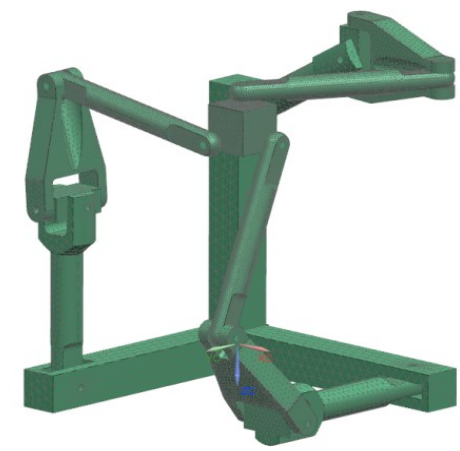

b)

Fig. 2. FEM model of the considered R-CUBE haptic device: a) Test Pose 1 (TP1); b) Test Pose 2 (TP2). 
Table 3 . shows the values $\lambda_{\mathrm{i}}(\mathrm{i}=1, \ldots, 10)$ of the first ten eigenvalues that are computed for four test poses TP1, TP2, TP3 and TP4 whose joint configuration is described in Table 4 . The stiffness performance can be locally analyzed by referring to the maximum and minimum eigenvalues of the stiffness matrix as they provide information on the maximum and minimum deformations at a given pose, [11]. Moreover, the simulation results in Table 3 show that the minimum value of the eigenvalues of each test pose is similar to the others. On the other hand, a difference can be identified in terms of maximum eigenvalues. In fact, TP1 shows a maximum natural frequency of $653.37 \mathrm{~Hz}$ that is the smallest one among the four simulated configurations. The FE simulations are also used to address the safety aspect that is due to accidental collisions between the end-effector of the manipulator and an operator.

Table 2. FEA computed compliant displacements under only gravity or gravity plus a load of $1.81 \mathrm{~N}$ that is applied along X-, Y- or Z-directions.

\begin{tabular}{ccccc}
\hline & \multicolumn{2}{c}{ TP1 } & \multicolumn{2}{c}{ TP2 } \\
\cline { 2 - 5 } & Force + Gravity & Gravity & Force + Gravity & Gravity \\
\hline$\Delta \mathrm{x}[\mathrm{mm}]$ & 0.054 & 0.001 & 0.164 & 0.006 \\
$\Delta \mathrm{y}[\mathrm{mm}]$ & 0.057 & 0.004 & 0.148 & 0.009 \\
$\Delta \mathrm{z}[\mathrm{mm}]$ & 0.071 & 0.013 & 0.114 & 0.017 \\
\hline
\end{tabular}

Table 3. FEA simulated first 10 natural frequencies at test poses TP1, TP2, TP3 and TP4.

\begin{tabular}{crrrr}
\hline & \multicolumn{1}{c}{ TP1 } & \multicolumn{1}{c}{ TP2 } & \multicolumn{1}{c}{ TP3 } & \multicolumn{1}{c}{ TP4 } \\
\hline$\lambda_{1}[\mathrm{~Hz}]$ & 96.19 & 98.25 & 95.55 & 96.79 \\
$\lambda_{2}[\mathrm{~Hz}]$ & 125.12 & 101.75 & 103.23 & 108.25 \\
$\lambda_{3}[\mathrm{~Hz}]$ & 131.67 & 135.33 & 144.42 & 142.15 \\
$\lambda_{4}[\mathrm{~Hz}]$ & 175.67 & 214.08 & 221.65 & 206.63 \\
$\lambda_{5}[\mathrm{~Hz}]$ & 189.29 & 227.04 & 231.59 & 233.25 \\
$\lambda_{6}[\mathrm{~Hz}]$ & 285.54 & 248.97 & 248.02 & 248.08 \\
$\lambda_{7}[\mathrm{~Hz}]$ & 343.71 & 356.16 & 349.15 & 342.72 \\
$\lambda_{8}[\mathrm{~Hz}]$ & 439.15 & 419.22 & 414.35 & 409.63 \\
$\lambda_{9}[\mathrm{~Hz}]$ & 503.22 & 698.75 & 682.64 & 616.14 \\
$\lambda_{10}[\mathrm{~Hz}]$ & 653.37 & 725.43 & 716.71 & 658.26 \\
\hline
\end{tabular}

Table 4. Joint configuration of the selected testing poses.

\begin{tabular}{ccccc}
\hline Joint Configuration [deg] & TP1 & TP2 & TP3 & TP4 \\
\hline$\phi_{11}$ & -30 & 0 & 0 & 0 \\
$\phi_{21}$ & -30 & 0 & +30 & -30 \\
$\phi_{31}$ & -30 & +30 & +30 & +30 \\
\hline
\end{tabular}


It is to note that the main mechanical risk cases for a robot can be identified as a direct collision with humans or indirect collisions where a human can be hit by elements that a robot hurls or drops. The latter case is not being expected for a haptic device. A possible way to describe the safety reliability of a service robot can be based on [13]. In particular, the safety performance of the R-CUBE can be assessed by computing an index that considers the acceleration of the impact between the device and the head of an operator. The Safety Index for Robots (SIR), [13], relies on the definition of safety used in automotive industry such as the widely used HIC (Head Injury Criterion) index, [13]. Considering the case of the R-CUBE end-effector moving at uniform velocity $v$, it is possible to assume the system robot-operator as a mass-spring-mass model, with $\mathrm{K}$ being the combined stiffness of the R-CUBE and the human head, $M_{r}$ the mass of R-CUBE and $M_{o p e r}$ the impacted operator-head mass, [13]. Thus, the SIR index can be computed as

$$
S I R=2560 \times(2 / \pi)^{\frac{3}{2}}(1 / 9.81)^{\frac{5}{2}}\left(K / M_{\text {oper }}\right)^{\frac{3}{4}}\left(M_{r} /\left(M_{r}+M_{\text {oper }}\right)\right)^{\frac{7}{4}} v^{\frac{5}{2}}
$$

Considering that the stiffness of the manipulator changes with the pose, it is convenient to analyze the variability of the SIR values among the different configurations as well as different directions of impact. Table 5 shows the SIR values associated to four different testing poses along X-, Y- and Z-directions. A constant speed of $2 \mathrm{~m} / \mathrm{s}$ has been here assumed by referring to common operating conditions. Analyzing the obtained results, one can note that the SIR values associated to TP1 are larger than the others. Accordingly the first tested pose represents a safer configuration. On the other hand, the poses TP2, TP3 and TP4 have similar safety behaviors.

Table 5. Computed SIR values along three directions, for a constant speed of $2 \mathrm{~m} / \mathrm{s}$.

\begin{tabular}{ccccc}
\hline SIR & TP1 & TP2 & TP3 & TP4 \\
\hline SIR $_{\mathrm{X}}$ & 0.28 & 0.12 & 0.12 & 0.15 \\
SIR $_{\mathrm{Y}}$ & 0.27 & 0.13 & 0.14 & 0.12 \\
SIR $_{Z}$ & 0.23 & 0.16 & 0.13 & 0.19 \\
\hline
\end{tabular}

\section{Experimental validation}

A specific test rig has been developed for measuring the compliant displacements of a R-CUBE prototype, also by considering previous experiences as reported in [1417]. The proposed test rig includes a 3D printed R-CUBE prototype, mechanic stops to fix the structure in the desired pose, a laser sensor for measuring the compliant displacements, a set of pulleys and weights to apply a desired loading condition at a desired pose, a NI6009 USB National Instruments data acquisition board, and a dedicated LabView Virtual Instrument for data acquisition and processing. It is worth noting that the model of R-CUBE haptic device has been built with the same material 
that has been considered in the FEM analyses. Single components have been experimentally checked to verify that the material properties match with FEM. The proposed test rig can apply a specific load aligned along X-, Y- and Z- directions. The used laser sensor measures the corresponding compliant displacement along the direction of the applied load. The applied load is obtained by using cables and pulleys with calibrated weights of known mass. Namely, a mass of 185 grams is used to apply a force of $1.8 \mathrm{~N}$. Results are reported in Table 6.

The comparison of experimental and numerical results shows that the stiffness performance is significantly affected by the pose and loading conditions. Among the analyzed poses, TP1 shows the stiffest behavior. Also the safety performance (based on SIR index) shows TP1 as the most performing pose. On the other hand, TP4 shows the best performance in terms of modal shapes, since it has the biggest value of maximum natural frequency. The effect of the structural weight is negligible along $X$ - and $\mathrm{Y}$ - directions while it shows a maximum contribution of $18 \%$ along $\mathrm{Z}$ - direction as compared with the effect of a load of $1.81 \mathrm{~N}$. Comparing numerical and experimental results in Table 2 and 6, one notes that the structural stiffness is not the most significant source of compliance with compliant displacements due to structural stiffness being even more than $95 \%$ lower than overall measured compliant displacements. The above effects prove that joint stiffness and clearance, as well as loading conditions and pose play the key role in the overall stiffness and safety performance.

Table 6. Experimental results of compliant displacements at the selected testing poses.

\begin{tabular}{ccc}
\hline & TP1 & TP2 \\
\cline { 2 - 3 } & Force + Gravity & Force + Gravity \\
\hline$\Delta \mathrm{x}[\mathrm{mm}]$ & 0.340 & 1.020 \\
$\Delta \mathrm{y}[\mathrm{mm}]$ & 0.450 & 0.930 \\
$\Delta \mathrm{z}[\mathrm{mm}]$ & 0.230 & 1.700 \\
\hline
\end{tabular}

Acknowledgments The paper presents results from the research activities of the project ID 37_215, MySMIS code 103415 "Innovative approaches regarding the rehabilitation and assistive robotics for healthy ageing" co-financed by the European Regional Development Fund through the Competitiveness Operational Programme 2014-2020, Priority Axis 1, Action 1.1.4, through the financing contract 20/01.09.2016, between the Technical University of Cluj-Napoca and ANCSI as Intermediary Organism in the name and for the Ministry of European Funds. 


\section{Conclusions}

This paper addresses the significance of structural properties on stiffness and safety of a R-CUBE Haptic Device. Namely, FEM analysis is used to compute indices of the performance of a R-CUBE prototype such as maximum compliant displacements, the minimum eigenvalues, and safety index SIR at four test poses. Results are also compared with evidences from experimental tests. This gives the possibility to highlight the contribution of structural properties on overall stiffness and safety performance as well as the significance of loading conditions, pose, and joint stiffness/clearance.

\section{References}

1. Hayward V., Astley O.R., et al. (2004). Haptic interfaces and devices. Sensor Review 24 (1):16-29.

2. Okamura A.M., "Haptic feedback in robot-assisted minimally invasive surgery", Current opinion in urology, vol. 19, no. 1, p. 102, 2009.

3. Salisbury, J. K., Srinivasan, A. M., "Projects in VR. Phantom - Based Haptic Interaction with Virtual Objects," IEEE Computer Graphics and Applications, pp. 6-10, 1997.

4. ISO 14121-1. Safety of machinery. Risk assessment. Part 1: principles; 2007.

5. EN ISO 13855. Safety of machinery - the positioning of protective equipment in respect of approach speeds of parts of the human body; 2010.

6. ISO/DIS 13482. Robots and robotic devices - safety requirements for non-industrial robots non-medical personal care robot; 2011.

7. Li, W., Gao, F., Zhang, J.: R-cube, a decoupled parallel manipulator only with revolute joints. Mechanism and Machine Theory 40(4), pp.467-473, 2005.

8. Takeda Y., Kamiyama K., Maki Y., Higuchi M., Sugimoto, K., "Development of positionorientation decoupled spatial in-parallel actuated mechanisms with six degrees of freedom," Journal of Robotics and Mechatronics, vol. 17, No. 1, pp. 59-62, 2005.

9. Biligincan T., Can Dede M.I., "Development of a R-CUBE-Based General Purpose Haptic Device System", ASME 2010 10th Biennial Conference on Engineering Systems Design and Analysis, Istanbul, pp. 675-682. DOI: 10.1115/ESDA2010-24596, 2010.

10. Gorgulu, I., Dede, M. I., „Computation time efficient stiffness analysis of the modified R-CUBE mechanism“, Int.Conference of IFToMM ITALY, pp.231-239. Springer, 2018.

11. Carbone G., "Stiffness analysis and experimental validation of robotic systems", Frontiers of Mechanical Engineering 6(2), pp.182-196, 2011.

12. Hibbeler R.C., "Mechanics of Materials", Pearson, London, 2014.

13. Cordero C.A., Carbone G., Ceccarelli M., Echavarri J., Munoz J.L., "Experimental tests in human-robot collision evaluation and characterization of a new safety index for robot operation", Mechanism and Machine Theory, Vol. 80, Pp.184-199, 2014.

14. Carbone G., Ceccarelli M., "Experimental Tests on Feasible Operation of a Finger Mechanism in the LARM Hand", Mech. Based Des. of Struct. and Mach., Vol.36, pp.1-13, 2008.

15. Carbone G., Iannone S., Ceccarelli M., "Regulation and Control of LARM Hand III", Rob. and Comp. Int. Manu., Vol.26, pp.202-211, 2010.

16. Hernández-Martínez E.E., Ceccarelli M., Carbone G., López-Cajún C.S., Jáuregui-Correa J.C., "Characterization of a Cable-Based Parallel Mechanism for Measurement Purposes", Mechanics Based Design of Structures and Machines, Volume 38, Issue 1, pp.25-49, 2010.

17. Major K.A., Major Z.Z., Carbone G., Pisla A., Vaida C., Gherman B., Pisla D.L., "Ranges of motion as basis for robot-assisted poststroke", Human and Veterinary Medicine, Vol.8, Issue 4, December 2016, Pages 192-196. 